\title{
CORRIGENDUM \\ Cost and Effectiveness of Combination Nicotine Replacement Therapy Among Heavy Smokers Contacting a Quitline-CORRIGENDUM
}

Laurie Krupski, K. Michael Cummings, Andrew Hyland, Martin C. Mahoney, Benjamin A. Toll, Matthew J. Carpenter, and Shannon Carlin-Menter

doi:10.1017/jsc.2014.15, Published online by Cambridge University Press, 30 June 2014.

An error has been noted in the above mentioned article by Krupski et al. In the discussion section in the fourth paragraph, 'greater than 15 cigarettes per day' should read 'greater than or equal to 10 cigarettes per day'.

The corrected sentence should read:

This finding mirrors the results of a recent randomised trial that found less of an effect of combination NRT for heavier smokers, defined as smoking greater than or equal to 10 cigarettes per day (Smith et al., 2013).

\section{Reference}

Krupski, L., Cummings, K.M., Hyland, A., Mahoney, M.C., Toll, B.A., Carpenter, M.J and Carlin-Menter, S. Cost and Effectiveness of Combination Nicotine Replacement Therapy Among Heavy Smokers Contacting a Quitline. Journal of Smoking Cessation, Published online 30 June 2014, doi:10.1017/jsc.2014.15 